\title{
Prevalence of Herbal Medicine Use among Pregnant Women in Zirobwe Health Centre III, Luwero District in Uganda.
}

\author{
Josephine Nabirye ${ }^{a, 1}$, Josephine Najjemba ${ }^{a}$
}

a School of Nursing and Midwifery, Aga Khan University

\begin{abstract}
\end{abstract}

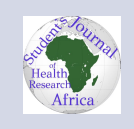

Background:

The prevalence of herbal medicine use is on the rise across the world, especially amongst pregnant women. The purpose of this study was to assess the prevalence of herbal medicine use among pregnant women in Zirobwe Health Center III, Luwero District in Uganda.

Methodology:

A cross-sectional research design was used on pregnant women aged between 18-38 years, selected using simple random sampling method and questionnaires to collect data which was analyzed using Statistical Package for the Social Sciences (SPSS).

Results:

All respondents 68(100\%) used herbal medicine during pregnancy, (58.8\%) used herbal medicine mostly in the first 3 months of the pregnancy, (100\%) sometimes used herbal medicine concurrently with conventional medicine, (97.1\%) never disclosed herbal medicine use to the physician/health care provider. The most common condition was nausea and vomiting $(95.5 \%)$, induce/enhance

labour (91.1\%), keep the baby healthy (88.3\%), abdominal/waist pain (85.2\%) and to boast or maintain health (73.5\%). Reasons for using herbal medicine during pregnancy were; parental/relative advice (88.3\%), safe during pregnancy $(85.2 \%)$, cheaper than conventional medicine $(73.5 \%)$, readily available $(70.5 \%)$, used when conventional medicine fails (58.8\%), complementing conventional medicine (47.1\%), more effective than conventional medicine (44.1\%), and being part of culture to use it (26.4\%).

Conclusion and recommendations: ${ }^{a}$

The use of herbal medicines in pregnancy was high and attention should be given to education of pregnant women and the community as a whole on the potentials if any and side effects of traditional medicine use during pregnancy.

${ }^{a}$ submitted: 19th/8/2021 accepted:
15th/9/2021 email: josephinek-
isakye27@gmail.com

\section{Background to the Study}

Herbal medicine (HM) has been used by humans for thousands of years including pregnant women (Oyebode et al., 2017). The World Health Organization (WHO) defines herbal medicines to include herbs, herbal materials, herbal preparations, and finished herbal products that contain as active in- gredients parts of plants, or other plant materials, or combinations (Will et al., 2009). Examples of herbal medicines used by women that are possibly unsafe during pregnancy include; Rosemary, garlic, saw palmetto, Goldenseal, Dong Quai, Ephedra, Yohimbe, sage, turmeric, ginger, Pay D'Arco, pas- 
sionflower,Black Cahosh, Roman Chamomile, and Pennynoyal among others. (Gorski et al., 2016).

Therefore, pregnant women are urged to consult a trained and experienced herbalist if they want to take herbs during their pregnancy. Some herbal products may contain agents that are contraindicated in pregnancy and may also have substances that can cause miscarriage, premature birth, uterine contractions or injury to the fetus.

However, some herbs are rated safe during pregnancy more especially when topically used such as Blond Psyllium, and Black Psylliun.

Pregnancy is a condition associated with immerse physiological alterations resulting in many pregnancy-related problems, including nausea, vomiting, constipation, and heartburn (Lindzon et al., 2015). These ailments usually result in pregnant women self-medicating using over-thecounter medications, seeking prescribed medications, or using herbs (Tabatabaee, 2015).

Some women may be advised by their families and friends to alleviate these uncomfortable changes such as heartburn, nausea, vomiting, and constipation by use herbs.

With the widespread use of various herbal products during pregnancy, herbal medicines have become an important issue in drug safety and clinical practice (Ekor, 2018). Even though evidence on the safety profile of native medicine products is inadequate to substantiate their use in pregnancy; it is increasingly used by expectant mothers.

While the use of herbal medicines in pregnancy varies considerably between countries, many of the same herbs are used. According to WHO estimates that the frequency of herbal medicine usage amongst pregnant women ranges between $7 \%$ and 96\% globally (Peprah et al., 2019). However, discrepancies exist in the consumption rate of herbal medicine during pregnancy between high resource and low resource economies which are largely accredited to cultural differences.

Evidence from the African continent suggests wide variability in the use of herbal medicines during pregnancy (Agyei-Baffour et al., 2017). In SubSaharan Africa, despite the heterogeneity and generally low quality of the identified literature, the review highlights the incidence of herbal medicine use among pregnant women to a tune of about 90\%representing a greater prevalence of herbs used in Africa than in the western world (James et al., 2018).
In Uganda, traditional herbal medicines are commonly used to prevent and treat a range of ailments during pregnancy (Tabuti et al., 2015). It is estimated that over $60 \%$ of the population including pregnant women use herbal medicine for day-to-day health needs, a pattern that cuts across all social classes and education levels(Nyeko et al., 2016).

(Nyeko et al., 2016) mentioned that While pregnant women recognize the potential risks of drug usage during pregnancy, they do not realize that herbal products could also be toxic, premised on the implicit belief that herbal products, being natural, are necessarily safe (Nikolajsen et al., 2015). This study sought to determine the prevalence, conditions prompting HM use, and factors contributing to herbal medicine use among pregnant women in Zirobwe Health Center III, Luweero District.

\section{Study Design \\ 2 Methodology}

The study adopted a cross-sectional design and employed a quantitative data collection approach. The choice of this design was due to its advantage to facilitate the collection of original data necessary to address the research objectives. It was useful in collecting data that could be quantified for reporting the true picture of the situation in the district.

\section{Study Setting}

The study was conducted in Zirobwe Health Center III. It is located in Luwero District approximately $41 \mathrm{~km}$ from Kampala. It is a public and major health facility offering healthcare services to the population of Zirobwe and neighboring areas. The facility offers services including in-patient and out-patient services, maternal and child health, HIV/AIDS, tuberculosis treatment, among others. The health center was preferred for this study because of the reported increase in herbal medicine use among pregnant women.

\section{Study Population}

This was divided into two; target and accessible population.

The target population was pregnant women.

The accessible population was pregnant women at Zirobwe Health Center III, Luweero District.

\section{Sample Size Determination}

The sample size was determined using Kish Leslie formula of 1965 for a single proportion.

$n=$ 
Where: $\mathrm{n}$ is the sample size

maximum likely error (taken to be 0.1 ).

is the standard deviation corresponding to $90 \%$ and a confidence interval of 1645

$P$ is the prevalence and $50 \%$ was assumed for this study in absence of actual statistics.

And $q$ was $1-p=1-0.5=0.5$

Thus, $\mathrm{n}=(1.645 \times 1.645)(0.5 \times 0.5)$

$0.1 \times 0.1$

$\mathrm{n}=(2.706025)(0.25)$

0.01

$\mathrm{n}=0.6760625$

0.01

Therefore, $n=68$ respondents.

\section{Sampling Procedure}

To select the respondents, a simple random sample technique was used. In this case, the ANC attendance cards of the pregnant women were arranged in the order in which the pregnant women were coming to the clinic and were picked at random by numbering the cards. Cards bearing the odd numbers that were; one, three, and five in that order were picked until the day was over. The daily attendance ranged from ten to twenty pregnant women. This was repeated at the ANC clinic in Zirobwe Health Center IIIto collect data on 68respondents.

\section{Inclusion Criteria}

Pregnant women aged $18-45$ years at the ANC clinic in Zirobwe Health Center III during the period of data collection, in good health and willing to participate were included in the study.

\section{Exclusion Criteria}

Pregnant women aged $18-45$ years at the ANC clinic but in poor health, the deaf, and those unwilling to participate were excluded from the study.

\section{Definition of Variables}

The variables assessed and measured included the following:

\section{Independent variables}

Demographic characteristics, a common condition for which HM is used, and factors contributing to herbal medicine use among pregnant women.

\section{Dependent variable}

Herbal medicine use during pregnancy.

Research Instrument

Data was collected using a questionnaire with questions written systematically in sections according to the study objectives. The questionnaire was designed in English, but the questions were asked in the local dialect which was Luganda for better understanding by illiterate respondents. The questionnaire was used to subject respondents to the same set of questions in a pre-determined order and it made data collection easier and faster. The questionnaires were administered to the respondents by the researchers themselves.

\section{Data Collection Procedure}

All the respondents signed a letter of consent (Appendix I) indicating their willingness to participate in this study. They were assured of confidentiality, the purpose of study, the potential benefits, and possible risks associated with participation were explained to them. The questionnaires for respondents (Appendix II) were interview-administered for those who were not literate enough and selfadministered for those who were literate.

\section{Quality Control}

To achieve this, a well-designed structured questionnaire containing all the details necessary to achieve the set objectives by obtaining the right information from pregnant women was used. Before the collection of data, investigators oriented themselves on data collection. The investigators undertook fieldwork. Completed questionnaires were checked for correctness and completeness. One independent person entered the data with the help of the investigators and the output was checked to ensure accuracy. A daily review of work was done and emerging problems were immediately addressed.

\section{Reliability and Validity}

The questionnaire was piloted among the pregnant women at Bamunanika Health Center, one of the health care centers in the district with similar characteristics with the study facility. The questionnaire was fine-tuned based on the responses from the pilot study before the main data collection.

\section{Data Management}

Data collected was first checked for completeness, then captured in a Microsoft Excel spreadsheet for data cleaning and coding. Data wasalso checked for consistency and inconsistent values. Numerical data were grouped for analysis purposes. Questionnaires judged to be incorrectly filled were rejected. Data was kept in a cabinet under lock and key to protect the respondents and to ensure confidentiality of the data collected. 


\section{Data Analysis and \\ Presentation}

Data analysis was undertaken using descriptive statistics, including frequencies. A software program called SPSS was employed for this study's data analysis. Frequencies for each variable were generated and organized into tables using SPSS. The results were interpreted and presented in a table and figures.

\section{Ethical Considerations}

Ethical approval was obtained from Aga Khan University Research and Ethics Committee. Permission was sought from the District Health Officer of Luwero. In addition, permission was sought and obtained from the appropriate authorities at Zirobwe Health Center III before the study commenced. Written consent was sought from respondents after a detailed explanation of what the study entailed was rendered. For confidentiality, respondents were required to answer the questionnaire without their names being written on the questionnaire for honest responses. Participation was voluntary and the pregnant women were told they could terminate answering the questionnaires at any time without any penalty.

\section{Limitations of the Study}

The study was conducted among pregnant women attending ANC clinics which may affect its generalizability since only a small proportion of women may attend ANC care. Similarly, the exclusion of emancipated minors who were not accompanied by adults was also likely to affect the generalizability of the study findings. This study also included only one lower health facility which may not be representative of the many health facilities at that level.

Information bias wasalso a likely problem since the use of herbal medicines is very often perceived negatively by health workers, and hence some women might have feared admitting to the use of herbal medicines.

Another limitation of the study was that no record was taken of the outcome of previous pregnancies and the researchers did not follow up the current pregnancy to assess the likely effects of herbal medicine being used by the women. Further study in this area should be designed to follow up women on herbal remedies to generate further knowledge on the safety of these herbs.

\section{Dissemination of Results}

The study results were compiled in a report and disseminated to Aga Khan University, District Health Officer, Luwero, Administration of Zirobwe Health Center III, and the Supervisor.

results are presented as obtained from the field. It handles sub-section under; socio-demographic data, conditions prompting herbal medicine use and factors contributing to herbal medicine use among pregnant women.

\section{Socio-Demographic Data}

\section{Table 1: showing Socio-Demographic Data}

Slightly more than half, 35 (51.5\%) of the respondents were aged between 18-24 years and the minority 13 (19.1\%) were aged between 32-38 years. Of the two age groups, respondents aged between 18-24 years used herbal more than those aged between 32-38 which reflected that they were more influenced to use herbs during pregnancy.

The majority 50 (73.5\%) of the respondents were married/cohabiting and they used herbal medicine than the rest of the respondents. The minority 8 (11.8\%) were divorced/separated.

More than half 38 (55.8\%) of the respondents attained primary level and the least 1 (1.5\%) had no formal education. Those who attained primary level of education used herbal medicine more frequently than the formal. However, $100 \%$ of the pregnant women used herbal medicine regardless of their age group, marital status, education level, occupation, and religion.

\section{Figure 1: Showing respondents' occupation}

$\mathrm{n}=68$

Majority 52(76.4\%) of the respondents were peasants and the least $1(1.5 \%)$ waitress.

Majority $45(66.2 \%)$ of the respondents were Christians while 23(33.8\%) were Moslem. 
Table 1. Showing Socio-Demographic Data

Variables
Age (Years)
$18-24$
$24-31$
$32-38$

$\begin{array}{ll}\text { Frequency } & \% \\ 35 & 51.5 \\ 20 & 29.4 \\ 13 & 19.1\end{array}$

Marital status

Single

Married/cohabiting

Divorced/separated

\section{Education level}

Primary

Secondary

None

\section{Religion}

Christianity

Islam

\section{Occupation}

Peasant

Small business

Waitress

Herbal medicine use during pregnancy

Yes

No

\section{Period when herbal medicine was used in pregnancy}

$1-3$

3-6

6-9

Throughout pregnancy

Use of herbal medicine together with conventional medicine

\section{Disclosure of herbal medicine to physician}

Yes

Physician's response in relation to respondents herbal medicine use

Discouraged

Encouraged Indifferent

\section{Person who recommended herbal medicine that was used during pregnancy}

Self

$\begin{array}{ll}0 & 0.0\end{array}$

Family friends 


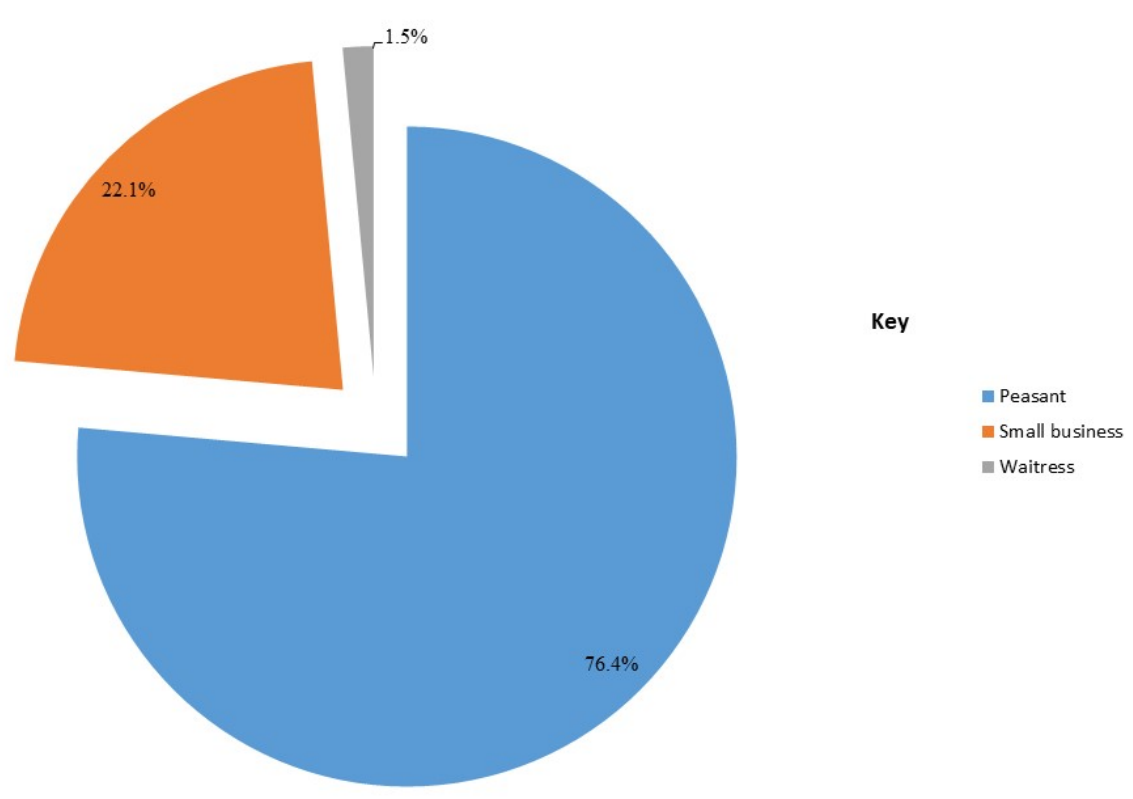

Chart 1. Showing respondents' occupation

\section{Figure 2: Showing number of pregnancies respondents have had before.}

$\mathrm{n}=68$

A larger majority 60 (88.3\%) of the respondents had two or more pregnancies while 8 (11.7\%) were carrying their first pregnancy.

\section{Prevalence of Herbal Medicine Use by Pregnant Mothers.}

All respondents 68 (100\%) used an herbal medicine during pregnancy.

More than half 40 (58.8\%) of the respondents used herbal medicine mostly in the first 3 months of the pregnancy while the remainder $2(2.9 \%)$ most pregnant women used herbal medicine between 3-6 months of the pregnancy.

The table above 4.2 , shows that respondents $68(100 \%)$ sometimes use herbal medicine concurrently with conventional medicine.

A larger majority 66 (97.1\%) of the respondents never disclosed herbal medicine use to the physician/health care provider while 2 (2.9\%) disclosed herbal medicine used to the physician.
Of the 2 respondents who disclosed herbal medicine use during pregnancy to the physician, $1(50 \%)$ of them was discouraged while 1 (50\%) was encouraged.

All respondents 68 (100\%) used an herbal medicine during pregnancy on the recommendations of family and friends.

\section{Common Conditions Prompting Herbal Medicine Use in Pregnancy}

Several ailments were listed for which herbal medicine was used during pregnancy. The most common condition was nausea and vomiting presenting 65 (95.5\%), followed by inducing/enhance labor 62 (91.1\%), keep the baby healthy 60 (88.3\%), abdominal/waist pain $58(85.2 \%)$, and boast or maintain health 50 (73.5\%). The fact that most pregnant women experience minor disorders during pregnancy, they believe herbal medicine works for them to relieve them from distress.

Factors Contributing to Use of Herbal Medicine by Pregnant Mothers

According to the analysis made, the reasons for using herbal medicine during pregnancy were; parental/relative advice presenting 60 (88.3\%) of respondents followed by safe during pregnancy with $58(85.2 \%)$ respondents, cheaper than conventional medicine 50 (73.5\%). The fourth reason was being readily available $48(70.5 \%)$, the fifth was used when conventional medicine fails $40(58.8 \%)$, fol- 


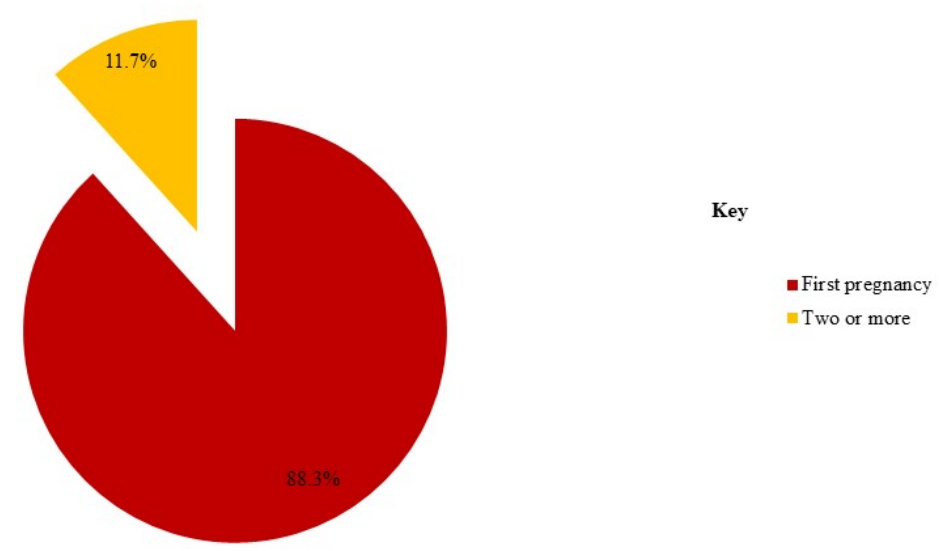

Chart 2. Showing number of pregnancies respondents have had before.

lowed by complementing conventional medicine 32 $(47.1 \%)$, more effective than conventional medicine $30(44.1 \%)$, and being part of the culture to use it $18(26.4 \%)$.

The next chapter is 5 and it discusses the results, conclusion, and recommendations.

\section{DISCUSSION, CONCLUSION AND RECOMMENDATIONS}

\section{Discussion}

\section{Socio-Demographic Data}

The socio-demographic data of the respondents is discussed in general terms and not about herbal medicine use during pregnancy as no relationship was sought in this case.

Slightly more than half, $51.5 \%$ of the respondents were aged between 18-24 years. This is because these years lie within the reproductive age group that is so active where these women are expected to have children or pregnant. This was dissimilar to Al-Riyami et al. (2015) in Omani women where 60\% of the pregnant mothers studied ranged between 28-35 years.

The majority (73.5\%) of the respondents were married/ cohabiting. This may be because it is traditionally and socially acceptable for any woman who comes of age to marry and remain in that marriage. Alternatively, traditionally once girls become pregnant, they are encouraged to go to their husbands' place. This was in agreement with Alor (2015) in Agotime-Ziope District, Ghana who also revealed that $70 \%$ of the mothers studied were married/ cohabiting.

More than half (55.8\%) of the respondents attained the primary level. This shows that wider gaps exist in literacy levels between the urban and rural dwellers in Uganda. The education levels in rural areas remain low despite the government efforts to promote education for all. This was inconsistent with Mbwanji (2015) among pregnant women attending antenatal clinics at Mbeya Referral Hospital in Tanzania where $60 \%$ of them attained secondary level.

The majority (76.4\%) of the respondents were peasants. The explanation for this could be that most of the respondents had low levels of education and could therefore not secure other jobs with ease. In addition, it may be because the study was done in a rural setting with agriculture being the dominant economic activity. Dabaghianet al. (2015) reported that in a group of pregnant women in Tehran, results revealed that 50\% were involved in business activities.

The majority (66.2\%) of the respondents were Christians. This could be because Uganda is a predominantly Christian nation. However, this was 
contrary to the study by Al-Riyami et al. (2015) in Omani where the majority of the respondents were Islam.

A larger majority (88.3\%) of the respondents had had two or more pregnancies, which may be attributed to 18-38 years being within the reproductive age group that is so active where these women are expected to have children. Similarly, Nergardet al. (2015) in Mali found that $74 \%$ of the respondents had two or more children.

\section{Prevalence of Herbal Medicine Use by Preg- nant Mothers}

In this study, all respondents (100\%) used an herbal medicine during pregnancy. This may be attributed to the poor educational attaining of women in this study as less educated women comparatively use herbal medicine more than women with higher education because they are likely to know the side effects of herbal medicine usage during pregnancy. Results from the previous study from Mali, West Africa diverge from the percentage of women reporting having used an herbal medicine during pregnancy with $79.9 \%$ reporting such use (Nergard et al., 2015).

Herbal medicines in the study setting were mainly used during the first trimester (58.8\%) and throughout pregnancy (33.8\%). This probably is due to the higher incidence of pregnancy-related problems during this period. In addition, the variability in the timing of use of the herbal medicines could be explained by the differences in the perceived desired effects and the reasons for use at a particular time; socio-cultural variability and belief, as well as differences in the types of herbs used by the different population groups. This was in line with Lisha et al., (2015) in the Middle East where the majority of women used these products during their first trimester.

All respondents (100\%) sometimes used herbal medicine concurrently with conventional medicine. This finding might reflect a certain open lifestyle where both herbal and conventional medicine use is common. Similarly with this finding, Tabatabace (2015) in Valiasr Hospital in Kazeroon, Fars, South of Iran indicated that a significant number of the users of herbal medicines during pregnancy used the herbal products alongside the conventional medicines given during antenatal care. This finding has important policy implications it presents a serious concern to components of the goal-oriented antenatal care interventions, especially prevention of maternal anemia and malaria, as well as the elimination of mother-to-child transmission when HIV-positive expectant mothers do not adhere to antiretroviral drugs as a result of using herbal products.

A larger majority $(97.1 \%)$ of the respondents never disclosed herbal medicine use to the physician/health care provider. This might be due to high trust and reliance on traditional practitioners or potentially lack of communication between a midwife/doctor and pregnant women when it comes to the use of medicinal plants. Likewise in Iran, Sattari et al., (2015) showed that only (12.5\%) of the respondents had revealed such use to healthcare professionals.

All respondents (100\%) used a herbal medicine during pregnancy on the recommendations of family and friends. This may be because family and friends represent the social and cultural environment in which the pregnant woman lives and significantly influences what is used during pregnancy. Contrasting findings have been reported by Malan et al., (2015) in Eastern Côte d'Ivoire that in most cases the women used herbal medicine on their initiative.

Common Conditions Prompting Herbal Medicine Use in Pregnancy

In this study, several ailments/conditions were found to prompt herbal medicine during pregnancy, and nausea and vomiting were the conditions most commonly treated (95.5\%), followed by induce/enhance labor (91.1\%), keeping the baby healthy (88.3\%), abdominal/waist pain (85.2\%) and to boast or maintain health $(73.5 \%)$. If women with access to health care facilities choose to treat these conditions with herbal medicine as a replacement for conventional medicine or do concurrently use both, this may have severe negative consequences for pregnancy outcomes. In the same way, Al-Riyami et al., (2015) in Omani revealed that the majority of users of herbal medicines during pregnancy had multiple reasons for use of these medicines, including abdominal/ waist pain, febrile illness, inducing/enhancing labor, skin problems, nausea and vomiting among others. This contradicts with the ailments treated in Norway (Nordeng et al., 2015) where the most commonly reported indications for using traditional medicine during pregnancy by respondents were cold and respiratory tract illness, nutritional supplement, skin prob- 
lems, pregnancy-related problems, urinary tract infections, and central nervous system disorders.

Factors Contributing to Use of Herbal Medicine by Pregnant Mothers

According to the findings, the most common reason for using herbal medicine during pregnancy was parental/relative advice (88.3\%). This may be because in developing countries personal and social links influence health care and what is used especially for pregnant women. This was consistent with Dabaghian et al., (2015) in Tehran were $51.3 \%$ of the respondents stated that parental or relative advice was a major factor that influenced their use of herbal remedies.

Another reason for the use of herbal medicine during pregnancy as indicated in this study was that it is safe during pregnancy (85.2\%) as well as being cheaper than conventional medicine (73.5\%), which supported the previous findings by Nergard et al., (2015) in Mali, West-Africa that most the respondents believed that medicinal plants had no adverse effects for the mother therefore safe to use. This may be attributed to poor educational attainment because they are unlikely to know the side effects of traditional medicine used during pregnancy. Findings of another study in Nigeria indicated that educational status is an important determinant factor in the use of traditional medicine and other alternative systems of health care delivery (Tamuno et al., 2016).

Among the other reasons reported for herbal medicine use during pregnancy was it being readily available $(70.5 \%)$, used when conventional medicine fails (58.8\%) as well as complementing conventional medicine (47.1\%). This may explain wide gaps in terms of conventional medicine availability at the health facilities, especially in rural areas. Additionally, probably due to low education, many mothers tend to ignore many aspects related to herbal medicine use. correspondingly, Mothupi (2015) in Nairobi, Kenya, 19\% of the respondents used traditional medicine because it was always available when they needed it.

Also, (44.1\%) of the respondents used herbal medicine because of the belief that it is more effective than conventional medicine (44.1\%). This may probably be due to high trust and reliance on traditional practitioners. The belief on the efficacy of herbal remedies in managing problems during pregnancy was similar to those reported by Nyeko et al. (2016) in a study where the use of herbal medicines during pregnancy was a perception that herbal medicines are effective in treating many ailments.

Another factor mentioned for herbal medicine use by pregnant women was being part of the culture to use it $(26.4 \%)$. This may explain the fact that pregnant mothers in most areas of Africa have a strong attachment to their traditional medicine. Similarly, Alor (2015) in Agotime-Ziope District, Ghana indicated that (45\%) of the respondents used traditional medicine because it was part of their culture to use traditional medicines during pregnancy.

\section{Conclusion}

In this study, $100 \%$ of the sample population used herbal medicine in pregnancy. Pregnant women primarily used herbal medicine for various conditions/ailments ranging from nausea and vomiting, induce/enhance labor, keep the baby healthy, abdominal/waist pain and to boast or maintain health. The factors associated with the use of herbal medicines during pregnancy include parental/relative advice, beliefs in its safety, being cheaper and readily available, used when conventional medicine fails, complementing conventional medicine, more effective than conventional medicine, and being part of the culture to use it.

\section{Recommendations}

There is a strong need for community sensitization drives on the dangers of indiscriminate use of herbal medicine in pregnancy, as well as integration of trained traditional herbalists and all those community persons who influence the process in addressing the various health needs of pregnant women.

The common use of medicinal plants observed warrants special attention due to their unknown efficacy and risks. It seems important to establish a wider collaboration between the local communities, the traditional practitioners, and conventional health workers of the health care centers on the safe use of medicinal plants during pregnancy.

The study emphasized the widespread use and strong perception of herbal medicines by pregnant women, highlighting an urgent need for midwives, community health nurses, and health care practitioners to be aware of this practice and make efforts in obtaining information about the use of herbs by pregnant women. This will help to furnish women with information on the adverse effects they may have on the unborn fetus. 
Midwives and healthcare professionals should routinely include the herbal remedy category in the list of drug history when asking about the patient's drug use at booking. This will help to identify those who use herbal remedies and therefore assist them to take precautions relating to safety.

However, concerning culture, the safe herbs should be approved especially those used topically and have been positively reported effective by trained herbalists.

The tailored message should be developed to help pregnant women have high perceptions of risk to reduce the desire for traditional medicine use. Families and friends are major sources of motivation in the use of traditional medicine. It is therefore important to promote disinterest in the use of traditional medicine through media and women's groups. The District Health Directorate should consider these issues when designing interventions for mothers and pregnant women.

A recommendation is made for further research on a pharmacological study focusing on local commonly used herbal medicines. This should be done to identify the exact pharmacological compounds of herbs and evaluate the effects of these compounds on the foetus and pregnant women.

\section{Acknowledgment}

We would wish to acknowledge the support of our families and the assistance of the following persons.

I Josephine Najjemba, send a special debt of gratitude to my beloved husband Senfuma Livingstone, my children; Namubiru Mary, Sekatawa Anthony Savio, and Nkanji Adrian Medad.

Particular thanks go to the following faculties; Dr. Grace Edwards, Dr. Ahmed Sarki, and faculty Kyakuwaire Hellen for their guidance towards writing this research.

I want to appreciate my colleagues Nabirye Josephine, Bamukisa Eunice Winnie, Namayanja Gertrude, and the entire class of BSCM 2019 for the support rendered.

I Nabirye Josephine would like to extend my sincere appreciation to my mother Babita Rose, my brother Gwambuga Peter Taire, my sister Namakula Fatuma, my girls Khaitsa Divine and Kakai Shekainah. Special thanks also go to my faculty; Kyakuwaire Hellen, Dr. Grace Edwards, and Dr.
Ahmed Sarki for all the necessary assistance given to me while writing this research.

\section{A References:}

1. Abasiubong, F., Bassey, E. A., Udobang, J. A., Akinbami, O. S., Udoh, S. B., \&ldung, U. I. (2015). Self-medication: potential risks and hazards among pregnant women in Uyo, Nigeria. Pan Afr Med J.;13:15.

2. Agyei-Baffour, P., Kudolo, A., Quansah, D. Y., \&Boateng, D. (2017). Integrating herbal medicine into mainstream healthcare in Ghana: clients' acceptability, perceptions and disclosure of use. BMC Complementary and Alternative Medicine 17:513. https://doi.org/10.1186/s12906-017-20254 PMid:29191194 PMCid:PMC5709853

3. Alor, S. K. (2015). The use of traditional medicine among pregnant women in AgotimeZiope District, Ghana.

4. Al-Riyami, I. M., Al-Busaidy, I. Q., \&Al-Zakwani, I. S. (2015). Medication use during pregnancy in Omani women. Int J Clin Pharm;33(4):634641. https://doi.org/10.1007/s11096-011-9517-y PMid:21597985

5. Azriani, A. R., Siti, A. S., Zulkifli, A., Salleh H., Wan, W., \&Manaf, H. (2015). Women's attitude and socio-demographic characteristics influencing usage of herbal medicines during pregnancy in Tumpat district, Kelantan. Vol 40 No. 2.

6. Dabaghian, F. H., Fard, M. A., Shojaei, A., Kianbakht, S., Zafarghandi, N., \&Goushegir, A. (2015). Use and attitude on herbal medicine in a group of pregnant woman in Tehran. J Med Plants.;11(41):2233.

7. Ekor, M. (2018). The growing use of herbal medicines: issues relating to adverse reactions and challenges in monitoring safety. Review Article.

8. Gorski, D. H. \& Novella, S. P. (2016). Clinical trials of integrative medicine: testing whether magic works?" Trends in molecular medicine, vol. 20, no. 9, pp. 473-476. https://doi.org/10.1016/j.molmed.2 014.06.007 PMid:25150944

9. Hill, J., Kayentao, K., Achieng, F., Diarra, S., Dellicoour, S., Diawara, S. I., et al. (2015). Access and use of interventions to prevent and treat malaria among pregnant women in Kenya and Mali: a qualitative study.; doi:10.1371/journal.pone.0119848. https://doi.o rg/10.1371/journal.pone.0119848 PMid:25798847 PMCid:PMC4370378 
10. James, P. B., Wardle, J., \& Steel, A. (2018). Traditional, complementary and alternative medicine use in Sub-Saharan Africa: a systematic review. BMJ Glob Health;3:e000895. https://doi.org/1 0.1136/bmjgh-2018-000895 PMid:30483405 PMCid:PMC6231111

11. Kennedy, D. A., Lupattelli, A., Koren, G., \&Nordeng, H. (2015). Herbal medicine use in pregnancy: results of a multinational study. BMC Complementary and Alternative Medicine 13:355. https://doi.o rg/10.1186/1472-6882-13-355 PMid:24330413 PMCid:PMC4029224

12. Kish. L., John.W., \& Sons. (2012). Survey sampling slide share. https://www.slideshare.net/d rtamil/6-calculate-samplesize-cohort-studies?next _slideshow $=1$

13. Laelago, T., Yohannes, T., \&Lemango, F. (2016). Prevalence of herbal medicine use and associated factors among pregnant women attending antenatal care at public health facilities in Hossana town, Southern Ethiopia: facility based cross sectional study. Archives Public Health. ';74:7. https://do i.org/10.1186/s13690-016-0118-z PMid:26881052 PMCid:PMC4753650

14. Lindzon, G., Sadry, S., \& Sharp, J. (2015). Obstetric. In: Toronto notes for medical students. 27th Edition. Type \& Graphics Inc. Canada.

15. Lisha, J. J., \& Nisha, S. (2015). Herbal medicine use during pregnancy: A review from the Middle East. Oman Medical Journey, Vol. 30, No.4:229-236. https://doi.org/10.5001/omj.2015.4 8 PMid:26366255 PMCid:PMC4561638

16. Malan, D. F., \&Neuba, D. F. R. (2015). Traditional practices and medicinal plants use during pregnancy by Anyi-Ndenye women (Eastern Côte d'Ivoire). Afr J Reprod Health. ;15(1):85-93.

17. Mbwanji, J. (2015). Prevalence of herbal medicine use and associated factors among pregnant women attending antenatal clinic at Mbeya Referral Hospital in 2010. http://ir.muhas.ac.tz:808 0/jspui/handle/123456789/41.

18. Mothupi, M. C. (2015). Use of herbal medicine during pregnancy among women with access to public healthcare in Nairobi, Kenya: a cross-sectional survey. BMC Complementary and Alternative Medicine, 14:432. https://doi.or g/10.1186/1472-6882-14-432 PMid:25370478 PMCid:PMC4230355

19. Nergard, C. S., Ho, T. P. T., Diallo, D., Ballo, N., Paulsen, B. S., \&Nordeng, H. (2015). Attitudes and use of medicinal plants during pregnancy among women at health care centers in three regions of Mali, West-Africa. Journal of Ethnobiology and Ethnomedicine11:73. https://doi.org/10.1186/s13002 -015-0057-8 PMid:26453339 PMCid:PMC4600315

20. Nikolajsen, T., Nielsen, F., Rasch, V., Sorensen, P. H., Ismail, F., Kristiansen, U., et al. (2015). Uterine contraction induced by Tanzanian plants used to induce abortion. J Ethnopharmacol.;137(1):921-5. https://doi.org/10. 1016/j.jep.2011.05.026 PMid:21645605

21. Nyeko, R., Tumwesigye, N. M., \&Halage, A. A. (2016). Prevalence and factors associated with use of herbal medicines during pregnancy among women attending postnatal clinics in Gulu district, Northern Uganda. BMC Pregnancy and Childbirth 16:296. https://doi.org/10.1186/s12884-016-10955 PMid:27716105 PMCid:PMC5053208

22. Olowokere, A. E., \&Olajide, O. (2015). Women's perception of safety and utilization of herbal remedies during pregnancy in a local government area in Nigeria.Clinical Nursing Studies, Vol. 1, No. 4. https://doi.org/10.5430/cns.v1n4p9

23. Oyebode, O., Kandala, N., Chilton, P. J., \&Lilford, R. J. (2017). Use of traditional medicine in middle-income countries: a WHO-SAGE study. Health Policy and Planning, 984-991. https://doi .org/10.1093/heapol/czw022 PMid:27033366 PMCid:PMC5013777

24. Peprah, P., Agyemang-Duah, W., ArthurHolmes, F., Budu, H. I., Abalo, E. M., Okwei, R., et al. (2019). 'We are nothing without herbs': a story of herbal remedies use during pregnancy in rural Ghana. BMC complementary and alternative medicine, 19(1), 65. https://doi.org/10.1186/s1290 6-019-2476-x PMid:30876425 PMCid:PMC6419816

25. Sattari, M., Dilmaghanizadeh, M., Hamishehkar, H., \&Mashayekhi, S. O. (2015). Self-reported use and attitudes regarding herbal medicine safety during pregnancy in Iran.Jundishapur Journal of Natural Pharmaceutical Products. Vol 7(2): 45-49. https://doi.org/10.5812/jj npp.3416 PMid:24624153 PMCid:PMC3941856

26. Tabatabaee, M. (2015). Use of herbal medicine among pregnant women referring to Valiasr Hospital in Kazeroon, Fars, South of Iran. J Med Plants.; 10(37):96-108.

27. Tabuti, J. R. S., Kukunda, C. B., Kaweesi, D., \&Kasilo, O. M. J. (2015). Herbal medicine use in the districts of Nakapiripirit, Pallisa, Kanungu, and Mukono in Uganda. Journal of Ethnobiology and 
Ethnomedicine, 8:35. https://doi.org/10.1186/17464269-8-35 PMid:22943789 PMCid:PMC3484030

28. Will.S.,Hu (2009).chinese herbal medicine. ht tps://drhuclinic.com/chinese-herbal-medicine 